\title{
The role of vitamin C in pneumonia and COVID-19 infection in adults with European ancestry: a Mendelian randomisation
} study

\author{
L. L. Hui ${ }^{1,2}{ }^{\otimes}$, E. A. S. Nelson (D) ${ }^{1}$, S. L. Lin ${ }^{1}$ and J. V. Zhao (iD ${ }^{3 凶}$ \\ (c) The Author(s), under exclusive licence to Springer Nature Limited 2021
}

BACKGROUND: High dose vitamin C infusion has been proposed to treat critically ill patients, including patients with pneumonia and severe COVID-19. However, trials have shown mixed findings. Here we assessed the unconfounded associations of vitamin C with COVID-19 and pneumonia using the Mendelian randomisation approach.

METHODS: This is a separate-sample Mendelian randomisation study using publicly available data. We applied single nucleotide polymorphisms (SNPs) that were associated with plasma vitamin C, in a recent genome-wide association study (GWAS) as genetic instruments to the GWAS of severe COVID-19, COVID-19 hospitalisation and any infection in the COVID-19 host genetics initiative and the GWAS of pneumonia in the UK Biobank, to assess whether people with genetically predicted higher levels of plasma vitamin C had lower risk of severe COVID-19 and pneumonia.

RESULTS: Genetically predicted circulating levels of vitamin C was not associated with susceptibility to severe COVID-19, COVID-19 hospitalisation, any COVID-19 infection nor pneumonia. Similar results were obtained when a weighted median and MR-Egger methods were used.

CONCLUSIONS: Mendelian randomisation analysis provided little evidence for an association of genetically predicted circulating levels of vitamin C with COVID-19 or pneumonia and thus our findings provided little support to the use of vitamin C in prevention and treatment in these patients, unless high dose vitamin C infusion has therapeutic effects via different biological pathways.

European Journal of Clinical Nutrition (2022) 76:588-591; https://doi.org/10.1038/s41430-021-00993-4

\section{INTRODUCTION}

Mortality of severe pneumonia remains high in both developing and developed countries. With emerging virus infections, including COVID-19 that causes severe pneumonia, there is a call for more pragmatic and efficient designs to test different therapeutics both individually and in combination for treating pneumonia [1].

Use of vitamin C in the prevention and treatment of pneumonia has been proposed since the 1930s based on clinical observations of a favourable effect of vitamin $C$ on subjects with pneumonia in Germany [2, 3]. Large doses of vitamin $C$ through oral or intravenous administration were claimed to be beneficial in patients with viral pneumonia $[4,5]$ and appear to be safe [6]. It has been suggested that a dosage of $6 \mathrm{~g}$ of vitamin $C$ per day normalises leukocyte vitamin $C$ levels in respiratory infections [7] while $3-10 \mathrm{~g}$ per day results in lower overall mortality rates in critically ill patients [8]. During the COVID-19 pandemic, there has been advocacy for using antioxidants including vitamin $\mathrm{C}$ for prevention and treatment $[9,10]$.

Vitamin C may exert therapeutic effects on patients with severe pneumonia via various mechanisms. Vitamin C plays a key role in immune cell function including immune cell proliferation and differentiation. It is also a powerful antioxidant that scavenges reactive oxygen species and down regulates expression of proinflammatory modulators. Severe pneumonia triggers host inflammatory responses that impair gas exchange and contributes to sepsis and organ dysfunction. Vitamin $C$ mitigated oxidative stress and proinflammatory mediators in patients with severe community-acquired pneumonia [11].

Clinical trials have reported a reduction in mortality among patients with severe pneumonia [7, 12], and a lower severity of respiratory dysfunction [13-15] as well as a shorter stay in intensive care unit [16] in critically ill patients with vitamin C treatment compared to controls receiving placebo or standard care. However the most recent trial on patients in intensive care did not show improvements in organ failure, inflammation, and vascular injury with vitamin C treatment [17].

Currently a number of registered trials are examining the therapeutic effect of vitamin C administered orally or intravenously, alone or in combination with other supplements among COVID-19 patients in different countries. At least four small-scale

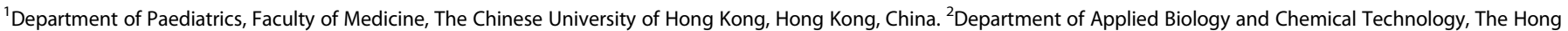

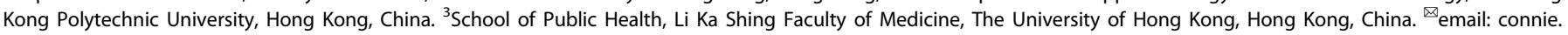
hui@polyu.edu.hk; janezhao@hku.hk 
trials have been published but the findings were mixed [18]. A RCT of 56 critical COVID-19 patients in China reported potential benefits of high dose vitamin $C$ infusion on oxygenation but similar 28-day mortality compared to controls [19]. Another small RCT in Iran $(n=60)$ observed some short term benefits, including lower body temperature and higher capillary oxygen saturations among severe COVID-19 patients treated with high dose vitamin C infusion, however there was no significant difference in $\mathrm{SpO}_{2}$ levels at discharge, the length of intensive care unit stay, and mortality between the two groups [20]. A larger RCT among 150 severe COVID-19 patients in Pakistan reported patients receiving vitamin $C$ became symptom-free earlier but did not use less mechanical ventilation [21]. Another RCT among milder COVID-19 patients in the US also showed that supplementation of vitamin C did not significantly decrease the duration of symptoms compared with standard care [22].

Mendelian randomisation study can be applied in nutritional epidemiology to clarify the etiologic role of specific nutrients. It uses single nucleotide polymorphisms (SNPs) to predict the dietary exposure and assess its relation with the health condition of interest. This technique has been increasingly utilised in assessing the effect of micronutrients on health, including vitamin B12 [23] and vitamin D [24]. Conventional observational studies assessing the role of diet in health are open to confounding as better diet is usually related to higher socioeconomic position, which is also associated with better health. In Mendelian randomisation studies, subjects are categorised by their genetic predisposition to the exposures of interest, which are not affected by confounders such as socioeconomic position. Therefore unlike conventional observational study, Mendelian randomisation analysis is not subject to confounding. Recent Mendelian randomisation studies have clarified that genetically determined Vitamin D exposure is not associated with COVID-19 infection and severity [25-27].

Here we assessed the associations of genetically predicted circulating levels of vitamin C with COVID-19 susceptibility/ severity and pneumonia with a two sample-Mendelian randomisation approach using publicly available data.

\section{METHODS}

\section{Genetic instruments for vitamin $C$}

We used SNPs that were associated with plasma vitamin $C$ level in the genome-wide association study (GWAS) in 52,018 people of European ancestry [28]. To check for their strength, we calculated the F-statistic; an Fstatistic $>10$ is considered to be a strong instrument.

\section{GWAS of COVID-19}

We used European-specific summary statistics using population as controls from the COVID-19 host genetics initiative (https://www.covid19hg.org/ results/) GWAS (Release 5), based on cohort studies mainly from Europe and the US [29]. We assessed three outcomes related to COVID-19:

Severe COVID-19 - defined as hospitalisation with laboratory confirmed SARS-CoV-2 infection as the primary reason for admission followed by death or respiratory support (cases $=4606$, non-cases $=702,801$ ).

COVID-19 hospitalisation - defined as hospitalisation with laboratory confirmed SARS-CoV-2 infection due to COVID-19-related symptoms (cases $=9373$, non-cases $=1,197,256$ )

COVID-19 infection - defined as laboratory confirmed SARS-CoV-2 infection (RNA and/or serology based), physician diagnosis of COVID-19, or self-report as COVID-19 positive (cases $=29,071$, non-cases $=1,559,712$ ). This group included severe COVID-19, COVID-19 hospitalisation and milder COVID-19 cases.

\section{GWAS of pneumonia}

We used publicly available summary statistics from the UK Biobank, with 14,054 cases of pneumonia among those with European ancestry downloaded from https://github.com/weizhouUMICH/SAIGE. This GWAS was adjusted for age, age squared, sex, the interaction of age and sex, and principal components.

\section{Statistical analysis}

To assess whether people with genetically predicted higher level of vitamin C had higher or lower risk of infection, we applied the genetic instruments to the selected GWAS and calculated the SNP-specific Wald estimates, i.e. the genetic association with severe COVID-19, COVID-19 hospitalisation, COVID-19 infection, and pneumonia, divided by the genetic association with vitamin C and then meta-analysed the SNPspecific Wald estimates using inverse variance weighting with multiplicative random effects.

In sensitivity analysis, we used different methods with different assumptions, including a weighted median and MR-Egger. A weighted median method can provide consistent estimates even when up to $50 \%$ of the information comes from invalid genetic variants [30]. MR Egger is able to detect potential pleiotropy from the significance of its intercept, as well as provide corrected estimates if such pleiotropy exists [31].

All statistical analyses were conducted using $R$ version 4.0.1 (R Foundation for Statistical Computing, Vienna, Austria), and the R package "MendelianRandomization". This analysis of publicly available data does not require ethical approval.

\section{RESULTS}

Nine SNPs for plasma levels of vitamin C were available in the GWAS of COVID-19 and pneumonia and were used (Supplementary Table 1), all with F-statistic $>10$. Genetically predicted circulating levels of vitamin $C$ were clearly not associated with severe COIVD-19, COVID-19 hospitalisation, any COVID-19 infection or pneumonia. (Table 1) These associations were robust to different analytic methods (Supplementary Table 2).

\section{DISCUSSION}

This is the first Mendelian randomisation study looking into the role of vitamin C in COVID-19 and pneumonia. Our findings suggested that genetically predicted circulating levels of vitamin $C$ was not associated with COVID-19 infection, its severity and pneumonia.

Despite decades of research, the therapeutic role of high dose vitamin C infusion in critically ill patients, including those with severe pneumonia, is still not clear. Our findings provided little evidence that normal vitamin $C$ levels play a role in susceptibility to COVID-19 and pneumonia and thus does not support the use of normal dose vitamin C for the prevention and treatment of COVID19 infection or pneumonia. Our null Mendelian randomisation association of vitamin C with severe COVID-19 and pneumonia was consistent with the mixed findings from recent vitamin $C$ trials on patients with severe COVID-19 published so far [19-22] and some previous trials among patients with pneumonia or critical conditions $[13,17]$. However, if the proposed therapeutic effects of high dose vitamin $C$ from either oral administration or intravenous infusion act via other biological pathways, our findings cannot exclude the possibility of a beneficial effect of high dose vitamin C infusion treatment in patients with severe COVID-19 or pneumonia.

Although this is a novel study using Mendelian randomisation technique, there are limitations and potential violations of key

Table 1. The Mendelian randomisation associations of genetically predicted circulating levels of vitamin C with COVID-19 infection and pneumonia.

\begin{tabular}{llll} 
Outcomes & OR & $\mathbf{9 5 \%} \mathbf{C l}$ & $\boldsymbol{P}$ value \\
\hline Severe COVID-19 & 1.00 & $0.74,1.35$ & 0.995 \\
\hline COVID-19 hospitalisation & 1.10 & $0.90,1.35$ & 0.35 \\
\hline Any COVID-19 infection & 1.04 & $0.93,1.15$ & 0.51 \\
\hline Pneumonia & 1.03 & $0.91,1.17$ & 0.61 \\
\hline
\end{tabular}

$O R$ odds ratio. 
assumptions, namely (1) strong instrument, (2) the SNP is not associated with confounders of the exposure-outcome associations and (3) the association between the SNPs and outcome occurs only through the hypothesised exposure. Genetic predictors capture a small proportion of the variance, making Mendelian randomisation estimates less biased but less precise, which may violate the strong instrument assumption and explain the wide confidence intervals. Measurement error might exist, however, we used genetic variants predicting plasma vitamin C level, rather than from questionnaire survey, which is less open to recall bias.

We cannot rule out violation of assumption that the SNPs and outcome occurs only through the hypothesised exposure. For example, asymptomatic individuals who are more health conscious may be more likely to have higher vitamin C intake and to be tested for COVID-19. However, getting a COVID-19 test is unlikely related to SNPs associated with vitamin $C$ and thus would not bias the directions of our results, but the possible measurement error in case ascertainment may make the estimates from Mendelian randomisation analysis more conservative. We are unable to rule out unknown pleiotropic effects potentially related to enzymes metabolising vitamin $C$ or chance finding. However the lead SNP that predicted plasma vitamin C level was involved in its metabolism, ruling out the potential limitations of the genetic instruments for vitamin $D$, which predicted the levels of bound vitamin $D$ instead of free vitamin D, in Mendelian randomisation analysis on COVID-19 infection [32]. Confounding by population stratification or a common data structure across studies is possible. However, we used GWAS consisting of people largely of European ancestry with genomic control while genetic associations with vitamin C and with COVID-19 were obtained from different studies. There are some ethnic groups having lower vitamin $C$ intake than Caucasians due to different cooking methods and dietary practice [33]. Given baseline vitamin C may influence the treatment effect of vitamin C [12], the findings from the Mendelian randomisation study based on European ancestry may not be applicable to other ethnic groups.

\section{CONCLUSION}

Mendelian randomisation analysis provided little evidence for an association of genetically predicted circulating levels of vitamin $C$ with COVID-19 or pneumonia, and thus does not support supplementing vitamin $\mathrm{C}$ in the prevention and treatment in these patients, unless high dose vitamin $C$ through either oral administration or intravenous infusion exerts a therapeutic effect via other biological pathways.

\section{REFERENCES}

1. Arabi YM, Fowler R, Hayden FG. Critical care management of adults with community-acquired severe respiratory viral infection. Intensive Care Med. 2020;46:315-28.

2. Bohnholtzer E. Contribution to the question of pneumonia treatment with vitamin C. Dtsch Med Wochenschr. 1937;63:1001-3.

3. Gander JN. Vitamin C in the treatment of pneumonia. Münch Med Wochenschr. 1936;83:2074-7. W.

4. Klenner FR. Massive doses of vitamin $C$ and the virus diseases. South Med Surg. 1951;113:101-7.

5. Cathcart RF. Vitamin C, titrating to bowel tolerance, anascorbemia, and acute induced scurvy. Med Hypotheses. 1981;7:1359-76.

6. Padayatty SJ, Sun AY, Chen Q, Espey MG, Drisko J, Levine M. Vitamin C: intravenous use by complementary and alternative medicine practitioners and adverse effects. PloS ONE. 2010;5:e11414.

7. Kim WY, Jo EJ, Eom JS, Mok J, Kim MH, Kim KU, et al. Combined vitamin C, hydrocortisone, and thiamine therapy for patients with severe pneumonia who were admitted to the intensive care unit: Propensity score-based analysis of a before-after cohort study. J Crit Care. 2018;47:211-8.

8. Wang Y, Lin H, Lin BW, Lin JD. Effects of different ascorbic acid doses on the mortality of critically ill patients: a meta-analysis. Ann Intensive Care. 2019;9:58.
9. Darenskaya M, Kolesnikova L, Kolesnikov S. The association of respiratory viruses with oxidative stress and antioxidants. Implications for the COVID-19 pandemic. Curr Pharm Des. 2021;27:1618-27.

10. Bogan-Brown K, Nkrumah-Elie $Y$, Ishtiaq $Y$, Redpath $P$, Shao A. Potential Efficacy of Nutrient Supplements for Treatment or Prevention of COVID-19. J Diet Suppl. 2021:1-29.

11. Chen Y, Luo G, Yuan J, Wang Y, Yang X, Wang $X$, et al. Vitamin $C$ mitigates oxidative stress and tumor necrosis factor-alpha in severe community-acquired pneumonia and LPS-induced macrophages. Mediators Inflamm. 2014;2014:426740.

12. Hunt C, Chakravorty NK, Annan G, Habibzadeh N, Schorah CJ. The clinical effects of vitamin $C$ supplementation in elderly hospitalised patients with acute respiratory infections. Int J Vitam Nutr Res. 1994;64:212-9.

13. Nathens AB, Neff MJ, Jurkovich GJ, Klotz P, Farver K, Ruzinski JT, et al. Randomized, prospective trial of antioxidant supplementation in critically ill surgical patients. Ann Surg. 2002;236:814-22.

14. Tanaka H, Matsuda T, Miyagantani $Y$, Yukioka T, Matsuda H, Shimazaki S. Reduction of resuscitation fluid volumes in severely burned patients using ascorbic acid administration: a randomized, prospective study. Arch Surg. 2000;135:326-31.

15. Fowler AA 3rd, Syed AA, Knowlson S, Sculthorpe R, Farthing D, DeWilde C, et al. Phase I safety trial of intravenous ascorbic acid in patients with severe sepsis. J Transl Med. 2014;12:32.

16. Hemila H, Chalker E. Vitamin C Can Shorten the Length of Stay in the ICU: a metaanalysis. Nutrients. 2019;11:708.

17. Fowler AA 3rd, Truwit JD, Hite RD, Morris PE, DeWilde C, Priday A, et al. Effect of Vitamin $C$ Infusion on Organ Failure and Biomarkers of Inflammation and Vascular Injury in Patients With Sepsis and Severe Acute Respiratory Failure: the CITRIS-ALI Randomized Clinical Trial. Jama. 2019;322:1261-70.

18. Milani GP, Macchi M, Guz-Mark A. Vitamin C in the Treatment of COVID-19. Nutrients. 2021;13:1172.

19. Zhang J, Rao X, Li Y, Zhu Y, Liu F, Guo G, et al. Pilot trial of high-dose vitamin C in critically ill COVID-19 patients. Ann Intensive Care. 2021;11:5.

20. JamaliMoghadamSiahkali S, Zarezade B, Koolaji S, SeyedAlinaghi S, Zendehdel A, Tabarestani $M$, et al. Safety and effectiveness of high-dose vitamin $C$ in patients with COVID-19: a randomized open-label clinical trial. Eur J Med Res. 2021;26:20.

21. Kumari P, Dembra S, Dembra P, Bhawna F, Gul A, Ali B, et al. The Role of Vitamin C as Adjuvant Therapy in COVID-19. Cureus. 2020;12:e11779.

22. Thomas S, Patel D, Bittel B, Wolski K, Wang Q, Kumar A, et al. Effect of High-Dose Zinc and Ascorbic Acid Supplementation vs Usual Care on Symptom Length and Reduction Among Ambulatory Patients With SARS-CoV-2 Infection: the COVID A to Z Randomized Clinical Trial. JAMA Netw Open. 2021;4:e210369.

23. Zhao JV, Schooling CM. Homocysteine-reducing B vitamins and ischemic heart disease: a separate-sample Mendelian randomization analysis. Eur J Clin Nutr. 2017;71:267-73.

24. Lu L, Bennett DA, Millwood IY, Parish S, McCarthy MI, Mahajan A, et al. Association of vitamin $D$ with risk of type 2 diabetes: a Mendelian randomisation study in European and Chinese adults. PLoS Med. 2018;15:e1002566.

25. Amin HA, Drenos F. No evidence that vitamin D is able to prevent or affect the severity of COVID-19 in individuals with European ancestry: a Mendelian randomisation study of open data. BMJ Nutr Prev Health. 2021;4:42-48.

26. Butler-Laporte G, Nakanishi T, Mooser V, Morrison DR, Abdullah T, Adeleye O, et al. Vitamin D and Covid-19 Susceptibility and Severity: a Mendelian Randomization Study. PLoS Med.2021;18:e1003605.

27. Patchen BK, Clark AG, Hancock DB, Gaddis N, Cassano PA. Genetically predicted serum vitamin D and COVID-19: a Mendelian randomization study. Curr Dev Nutr. 2021; 5(Suppl 2):1080.

28. Zheng JS, Luan J, Sofianopoulou E, Imamura F, Stewart ID, Day FR, et al. Plasma Vitamin C and Type 2 Diabetes: Genome-Wide Association Study and Mendelian Randomization Analysis in European Populations. Diabetes Care. 2021:44:98-106.

29. Initiative C-HG. The COVID-19 Host Genetics Initiative, a global initiative to elucidate the role of host genetic factors in susceptibility and severity of the SARSCoV-2 virus pandemic. Eur J Hum Genet. 2020;28:715-8.

30. Bowden J, Davey Smith G, Haycock PC, Burgess S. Consistent Estimation in Mendelian Randomization with Some Invalid Instruments Using a Weighted Median Estimator. Genet Epidemiol. 2016;40:304-14.

31. Burgess S, Thompson SG. Interpreting findings from Mendelian randomization using the MR-Egger method. Eur J Epidemiol. 2017;32:377-89.

32. Baah EK, M. When Mendelian randomisation fails. BMJ Nutr Prev Health. 2021;4:1-3.

33. Zhang R, Wang Z, Fei Y, Zhou B, Zheng S, Wang L, et al. The Difference in Nutrient Intakes between Chinese and Mediterranean, Japanese and American Diets. Nutrients. 2015;7:4661-88. 


\section{AUTHOR CONTRIBUTIONS}

All the authors contributed to the interpretation of the data, critically revising the paper and approval of the final version. Specifically and additionally, NEAS developed the study conception, LSL contributed to data analysis, HLL and ZJV directed the study's analytic strategy and wrote the paper.

\section{COMPETING INTERESTS}

The authors declare no competing interests.

\section{ADDITIONAL INFORMATION}

Supplementary information The online version contains supplementary material available at https://doi.org/10.1038/s41430-021-00993-4.

Correspondence and requests for materials should be addressed to L.L.H. or J.V.Z.

Reprints and permission information is available at http://www.nature.com/ reprints

Publisher's note Springer Nature remains neutral with regard to jurisdictional claims in published maps and institutional affiliations. 